\title{
Synthesis of Hexahydropyridazine-3-phosphonic Acid
}

\author{
Mamoru Kaname, Kazuo Yoshinaga, Yasushi Arakawa, and Shigeyuki Yoshifuji* \\ Faculty of Pharmaceutical Sciences, Hokuriku University; Kanagawa-machi Ho-3, Kanazawa 920-1181, Japan. \\ Received September 12, 2003; accepted October 6, 2003
}

The synthesis of hexahydropyridazine-3-phosphonic acid (piperidazine-3-phosphonic acid) was performed via a hetero-Diels-Alder reaction followed by Lewis acid-catalyzed phosphonylation. This two-step procedure was improved to a one-pot reaction.

Key words hexahydropyridazine-3-phosphonic acid; phosphonylation; one-pot reaction; piperidazine-3-phosphonic acid; $\alpha$-hydrazinophosphonic acid; hetero-Diels-Alder reaction

As analogues of $\alpha$-aminophosphonic acids, $\alpha$-hydrazinophosphonic acids (type 1, in Fig.1) and their derivatives are of potential biological importance. For example, several of these compounds provide safety against the phytotoxic action of chloroacetanilide herbicides. ${ }^{1)}$ A few examples of the synthesis of $\alpha$-hydrazinophosphonic acids have been reported; these include a nucleophilic phosphonylation to either preformed or in situ generated $\mathrm{C}=\mathrm{N}$ bonds (aliphatic aldehyde azines, ${ }^{2)} \mathrm{N}$-protected hydrazones, ${ }^{3)} \mathrm{di}-$ methylhydrazones ${ }^{4)}$, a selective reduction of $\alpha$-hydrazonophosphonic acids, ${ }^{5)}$ and a nucleophilic substitution of $\alpha$ mesyloxyalkylphosphonates with hydrazine. $\left.{ }^{6}\right)$ However, a simple and general synthetic method for cyclic hydrazinetype compounds (type 2) has not been established, and most of the reported methods concern acyclic compounds.

Recently, as a model of cyclic $\alpha$-hydrazinophosphonic acid, we reported the first synthesis of simple hexahydropyridazine-3-phosphonic acid (piperidazine-3-phosphonic acid) 3 in preliminary form, ${ }^{7)}$ employing the hetero-Diels-Alder (hetero-D-A) reaction and subsequent phosphonylation of the cycloadduct in the presence of a Lewis acid. Piperidazine-3-phosphonic acid is a phosphonic analogue of

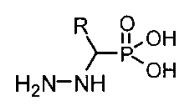

1

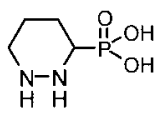

3

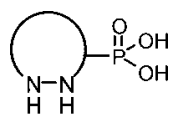

2

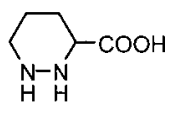

4 piperidazine-3-carboxylic acid 4 , the enantiomeric forms of which have been encountered in numerous pharmacologically active molecules, such as the monamycin ${ }^{8)}$ and azinothricin $^{9)}$ families. The details of the synthesis of $\mathbf{3}$ are described here.

In the initial stage, we examined the construction of the 3substituted pyridazine rings by the application of the reported methods ${ }^{10,11)}$ of the hetero-D-A reaction, as illustrated in Chart 1 . Thus the hetero-D-A reaction of 1-methoxy-1,3-butadiene 5a with dialkyl azodicarboxylates $\mathbf{6 a}-\mathbf{c}$ was carried out in dichloromethane $\left(\mathrm{CH}_{2} \mathrm{Cl}_{2}\right)$ at room temperature to produce the cycloadducts of 3-methoxy-1,2,3,6-tetrahydropyridazine derivatives $\mathbf{7 a}-\mathbf{c}$ in nearly quantitative yields. Conversion of the methoxyl group of the cycloadducts $\mathbf{7 a}-\mathbf{c}$ into a phosphonyl group was conducted smoothly in $\mathrm{CH}_{2} \mathrm{Cl}_{2}$ at room temperature by treatment of $7 \mathbf{a}-\mathbf{c}$ with trimethyl phosphite in the presence of trimethylsilyl triflate (TMSOTf) or boron trifluoride etherate $\left(\mathrm{BF}_{3} \cdot \mathrm{OEt}_{2}\right)$ as a Lewis acid to afford the corresponding methyl 1,2,3,6-tetrahydropyridazine-3-phosphonates $\mathbf{8 a -} \mathbf{c}$ in good yields. The results are summarized in Table 1. Despite these satisfactory results, a similar hetero-D-A reaction using trimethylsilyloxybutadiene $\mathbf{5 b}$ or acetoxybutadiene $\mathbf{5 c}$ was not quite as straightforward as expected. These cycloadducts are very unstable. Therefore our attention was directed toward a one-pot reaction including the hetero- $\mathrm{D}-\mathrm{A}$ reaction and subsequent phosphonylation. After some trials, the one-pot reaction progressed well by slowly adding a Lewis acid to the $\mathrm{CH}_{2} \mathrm{Cl}_{2}$ solution of three components (diene $\mathbf{5}$, dienophile $\mathbf{6}$, and trimethyl phospite) and gave $\mathbf{8 a}$ in satisfactory yields (Table 1 ), except for the case of acetoxybutadiene $\mathbf{5 c}$, in which the reaction became complicated. In comparison with the twostep method, this one-pot method appears to be advantageous in terms of reaction efficiency, particularly for the unstable cycloadducts from the hetero-D-A reaction.

Fig. 1

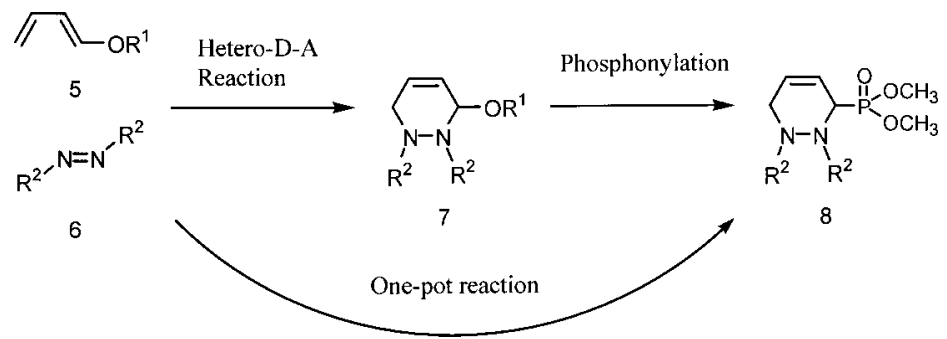

Chart 1 
Table 1. Hetero-D-A Reaction and Phosphonylation

\begin{tabular}{|c|c|c|c|c|c|c|c|c|}
\hline \multicolumn{2}{|c|}{$\begin{array}{l}\text { Diene } \\
\mathrm{R}^{1}\end{array}$} & \multicolumn{2}{|c|}{$\begin{array}{r}\text { Dienophile } \\
\mathrm{R}^{2}\end{array}$} & Lewis acid & \multicolumn{2}{|c|}{$\begin{array}{l}\text { Hetero-D-A } \\
\text { reaction } \\
\text { Yield }(\%)\end{array}$} & \multicolumn{2}{|c|}{$\begin{array}{c}\text { Phosphony- } \\
\text { lation } \\
\text { Yield (\%) }\end{array}$} \\
\hline \multirow[t]{3}{*}{$5 a$} & $\mathrm{Me}$ & $6 a$ & Boc & $\mathrm{BF}_{3} \cdot \mathrm{OEt}_{2}$ & $7 a$ & 99 & $8 \mathbf{a}$ & 81 \\
\hline & & $6 b$ & Troc & TMSOTf & $7 b$ & 98 & $8 b$ & 69 \\
\hline & & $6 c$ & Z & TMSOTf & $7 c$ & quant. & $8 c$ & 89 \\
\hline $5 b$ & TMS & $6 a$ & Boc & $\mathrm{BF}_{3} \cdot \mathrm{OEt}_{2}$ & $7 d$ & 64 & $8 \mathbf{a}$ & 99 \\
\hline $5 c$ & Ac & $6 a$ & Boc & & & -b) $^{-1}$ & & \\
\hline $5 a$ & $\mathrm{Me}$ & $6 a$ & Boc & $\mathrm{BF}_{3} \cdot \mathrm{OEt}_{2}$ & & & $8 \mathbf{a}$ & 96 \\
\hline $5 b$ & TMS & $6 a$ & Boc & $\mathrm{BF}_{3} \cdot \mathrm{OEt}_{2}$ & (one-p & t reaction) & $8 a$ & 99 \\
\hline $5 c$ & Ac & $6 a$ & Boc & $\mathrm{BF}_{3} \cdot \mathrm{OEt}_{2}$ & & & $8 \mathbf{a}$ & 19 \\
\hline
\end{tabular}

a) Boc, tert-butoxycarbonyl; Troc, trichloroethoxycarbonyl; Z, benzyloxycarbonyl. b) No product could be isolated.

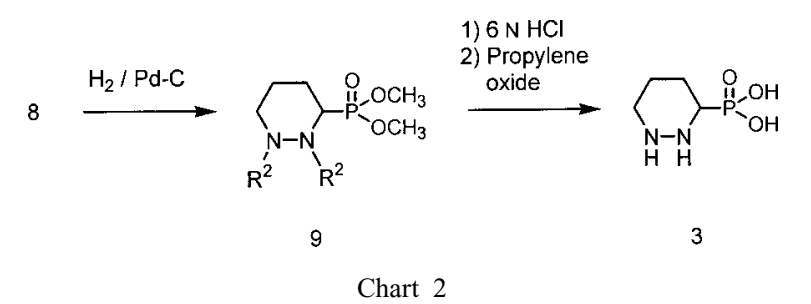

Next, catalytic hydrogenation of tetrahydropyridazines $\mathbf{8 a}$ and $\mathbf{8 c}$, which have tert-butoxycarbonyl (Boc) and trichloroethoxycarbonyl (Troc) groups, using Pd on charcoal in methanol gave the saturated compounds $9 \mathbf{a}$ and $\mathbf{9 b}$ in $89 \%$ and $40 \%$ yield, respectively, although the reduction of the Troc group ocurred in the latter. Finally, $N$-Boc derivative 9a was hydrolyzed in boiling $6 \mathrm{~N} \mathrm{HCl}$ and then treated with propylene oxide ${ }^{12)}$ in methanol to obtain the salt-free product to give piperidazine-3-phosphonic acid 3 in $62 \%$ yield (Chart 2 ), the structure of which was supported by the analytical and spectral data.

Thus the synthesis of piperidazine-3-phosphonic acid has been accomplished. The present method is simple and will be applicable to the synthesis of a variety of 6-membered cyclic $\alpha$-hydrazinophosphonic acids.

\section{Experimental}

All melting points were determined on a Yanagimoto micro melting point apparatus and are uncorrected. NMR spectra were obtained on a JEOL JNM-GSX-400 spectrometer using tetramethylsilane (TMS, $\delta 0 \mathrm{ppm}$ ) or dioxane ( $\delta 3.70 \mathrm{ppm}$ for ${ }^{1} \mathrm{H}$-NMR and $\delta 67.4 \mathrm{ppm}$ for $\left.{ }^{13} \mathrm{C}-\mathrm{NMR}\right)$ as an internal standard. IR spectra were recorded on a Horiba FT-720 spectrophotometer. MS and HR-MS were obtained on a JEOL JMS-SX102A spectrometer. Column chromatography was carried out on silica gel (Kieselgel 60, 70230 mesh, Merck). Dialkyl azodicarboxylates and 1-substituted 1,3-butadienes were purchased from commercial sources.

Di-tert-butyl 3-Methoxy-1,2,3,6-tetrahydropyridazine-1,2-dicarboxylate (7a) A solution of di-tert-butyl azodicarboxylate $\mathbf{6 a}(690 \mathrm{mg}, 3 \mathrm{mmol})$ in $\mathrm{CH}_{2} \mathrm{Cl}_{2}(3 \mathrm{ml})$ was stirred under cooling at $0{ }^{\circ} \mathrm{C}$. 1 -Methoxy-1,3-butadiene $5 \mathbf{a}(0.33 \mathrm{ml}, 3.3 \mathrm{mmol})$ was added to the solution. The reaction mixture was allowed to warm to room temperature, and stirring was continued for $2 \mathrm{~h}$. The reaction solution was evaporated in vacuo to leave a residue, which was purified by column chromatography $\left(\mathrm{CHCl}_{3}\right)$ to give $7 \mathbf{a}$ in $99 \%$ yield. Colorless oil. ${ }^{1} \mathrm{H}-\mathrm{NMR}\left(\mathrm{CDCl}_{3}\right) \delta: 1.49\left(9 \mathrm{H}, \mathrm{s}, \mathrm{C}_{4} \mathrm{H}_{9}\right), 1.50\left(9 \mathrm{H}, \mathrm{s}, \mathrm{C}_{4} \mathrm{H}_{9}\right), 3.49$ and $3.53^{13)}\left(3 \mathrm{H}\right.$, each $\left.\mathrm{s}, \mathrm{OCH}_{3}\right), 3.55-3.83(1 \mathrm{H}, \mathrm{m}, \mathrm{C} 6-\mathrm{Ha}), 4.31$ and $4.49^{13)}$ $(1 \mathrm{H}, \mathrm{d}, J=18.7 \mathrm{~Hz}$ and dd, $J=18.0,2.9 \mathrm{~Hz}, \mathrm{C} 6-\mathrm{Hb}), 5.25-5.66(1 \mathrm{H}, \mathrm{br}, \mathrm{C} 3-$ $\mathrm{H}), 5.77-6.15(2 \mathrm{H}, \mathrm{m}, \mathrm{C} 4-\mathrm{H}, \mathrm{C} 5-\mathrm{H}) .{ }^{13} \mathrm{C}-\mathrm{NMR}\left(\mathrm{CDCl}_{3}\right) \delta: 28.26,28.34$ (q), $41.68,43.64$ (t), 56.24 (q), 80.25 (d), 80.84, 81.01, 81.63 (s), 123.82, 124.33 (d), 127.21, 127.73 (d), 154.19, 154.48 (s). IR (neat) $\mathrm{cm}^{-1}: 1706$. MS m/z: $314\left(\mathrm{M}^{+}\right)$. HR-MS $m / z: 314.1842\left(\mathrm{M}^{+}\right)\left(\right.$Calcd for $\mathrm{C}_{15} \mathrm{H}_{26} \mathrm{~N}_{2} \mathrm{O}_{5}: 314.1842$ ).

The other cycloadducts $\mathbf{7 b}-\mathbf{d}$ were prepared in the same manner as described above. Yields of $\mathbf{7 b}-\mathbf{d}$ are summarized in Table 1.
Bis(2,2,2-trichloroethyl) 3-Methoxy-1,2,3,6-tetrahydropyridazine-1,2dicarboxylate (7b) Colorless oil. ${ }^{1} \mathrm{H}-\mathrm{NMR}\left(\mathrm{CDCl}_{3}\right) \quad \delta: 3.53(3 \mathrm{H}, \mathrm{s}$, $\left.\mathrm{OCH}_{3}\right), 3.81-3.96$ and $4.00-4.11(1 \mathrm{H}, \mathrm{m}, \mathrm{C} 6-\mathrm{Ha}), 4.53-5.05(5 \mathrm{H}, \mathrm{m}$, C6-Hb and $\left.\mathrm{COOCH}_{2} \times 2\right), 5.57(1 \mathrm{H}$, br s, C3-H), 5.88-6.09 $(2 \mathrm{H}, \mathrm{m}, \mathrm{C} 4-\mathrm{H}$, C5-H). ${ }^{13} \mathrm{C}-\mathrm{NMR}\left(\mathrm{CDCl}_{3}\right) \delta: 43.09,44.17(\mathrm{t}), 56.72,57.07(\mathrm{q}), 75.39,75.62$ (t), 81.18, $81.69(\mathrm{~d}), 94.72,94.85(\mathrm{~s}), 123.60,124.04(\mathrm{~d}), 126.41,126.88(\mathrm{~d})$, $152.67,153.17$ (s). IR (neat) $\mathrm{cm}^{-1}: 1728$. MS $m / z: 462\left(\mathrm{M}^{+}\right)$. HR-MS $m / z$ : $461.8884\left(\mathrm{M}^{+}\right)\left(\right.$Calcd for $\left.\mathrm{C}_{11} \mathrm{H}_{12} \mathrm{Cl}_{6} \mathrm{~N}_{2} \mathrm{O}_{5}: 461.8877\right)$.

Dibenzyl 3-Methoxy-1,2,3,6-tetrahydropyridazine-1,2-dicarboxylate (7c) Colorless oil. ${ }^{1} \mathrm{H}-\mathrm{NMR}\left(\mathrm{CDCl}_{3}\right) \delta: 3.28$ and $\left.3.57^{13}\right)(3 \mathrm{H}$, each brs, $\left.\mathrm{OCH}_{3}\right), 3.65-4.00(1 \mathrm{H}, \mathrm{m}, \mathrm{C} 6-\mathrm{Ha}), 4.41-4.62(1 \mathrm{H}, \mathrm{m}, \mathrm{C} 6-\mathrm{Hb}), 5.00-$ $5.35\left(4 \mathrm{H}, \mathrm{m}, \mathrm{COOCH}_{2} \times 2\right), 5.50-5.60(1 \mathrm{H}, \mathrm{br} \mathrm{s}, \mathrm{C} 3-\mathrm{H}), 5.81-5.99(2 \mathrm{H}$, $\mathrm{m}, \mathrm{C} 4-\mathrm{H}, \mathrm{C} 5-\mathrm{H}), 7.18-7.45\left(10 \mathrm{H}, \mathrm{m}, \mathrm{C}_{6} \mathrm{H}_{5} \times 2\right) .{ }^{13} \mathrm{C}-\mathrm{NMR}\left(\mathrm{CDCl}_{3}\right) \delta$ : $42.52,42.64(\mathrm{t}), 43.73,43.87(\mathrm{t}), 56.41(\mathrm{q}), 67.93,67.99,68.06,68.09(\mathrm{t})$, $80.78,80.90,81.09$ (d), $123.65,124.15$ (d), 127.34, 127.58 (d), 127.87, $127.97,128.11,128.25,128.32,128.43,128.55,128.58$ (d), 135.64, 135.87 (s), 154.40, 155.24 (s). IR (neat) $\mathrm{cm}^{-1}: 1716$. MS m/z: $382\left(\mathrm{M}^{+}\right)$. HR-MS $m / z: 382.1529\left(\mathrm{M}^{+}\right)\left(\right.$Calcd for $\left.\mathrm{C}_{21} \mathrm{H}_{22} \mathrm{~N}_{2} \mathrm{O}_{5}: 382.1529\right)$.

Di-tert-butyl 3-Trimethylsilyloxy-1,2,3,6-tetrahydropyridazine-1,2-dicarboxylate (7d) Colorless columns (hexane), mp 58-58.5 ${ }^{\circ} \mathrm{C} .{ }^{1} \mathrm{H}-\mathrm{NMR}$ $\left(\mathrm{CDCl}_{3}\right) \delta: 0.21(9 \mathrm{H}, \mathrm{s}, \mathrm{TMS}), 1.48\left(18 \mathrm{H}, \mathrm{s}, \mathrm{C}_{4} \mathrm{H}_{9} \times 2\right), 3.58$ and $3.72^{13)}(1 \mathrm{H}$, d, $J=17.8 \mathrm{~Hz}$ and d, $J=17.2 \mathrm{~Hz}, \mathrm{C} 6-\mathrm{Ha}), 4.29$ and $4.46^{13)}(1 \mathrm{H}, \mathrm{dd}, J=17.9$, $3.4 \mathrm{~Hz}$ and d, $J=17.2 \mathrm{~Hz}, \mathrm{C} 6-\mathrm{Hb}), 5.68-5.95(3 \mathrm{H}, \mathrm{m}, \mathrm{C} 3-\mathrm{H}, \mathrm{C} 4-\mathrm{H}, \mathrm{C} 5-\mathrm{H})$. ${ }^{13} \mathrm{C}-\mathrm{NMR}\left(\mathrm{CDCl}_{3}\right) \delta: 0.05(\mathrm{q}), 28.27,28.43(\mathrm{q}), 41.70,43.60(\mathrm{t}), 73.48$, 74.96 (d), 80.72, 81.45 (s), 125.63, 126.00, 126.45, 126.87 (d), 152.59, 153.48, 154.55, 154.64 (s). IR (KBr) cm ${ }^{-1}: 1697$. MS m/z: $372\left(\mathrm{M}^{+}\right)$. Anal. Calcd for $\mathrm{C}_{17} \mathrm{H}_{32} \mathrm{~N}_{2} \mathrm{O}_{5} \mathrm{Si}$ : C, 54.81; H, 8.66; N, 7.52. Found: C, 54.77; H, 8.49; N, 7.61.

Posphonylation of 7, General Procedure A solution of $7 \mathbf{a}-\mathbf{d}$ $(10 \mathrm{mmol})$ in $\mathrm{CH}_{2} \mathrm{Cl}_{2}(50 \mathrm{ml})$ was treated with trimethyl phosphite $(3.10 \mathrm{~g}$, $25 \mathrm{mmol})$ and TMSOTf or $\mathrm{BF}_{3} \cdot \mathrm{Et}_{2} \mathrm{O}(15 \mathrm{mmol})$ at $0{ }^{\circ} \mathrm{C}$. After $15 \mathrm{~min}$ the solution was allowed to warm to room temperature and was stirred for a further $12 \mathrm{~h}$. A saturated aqueous $\mathrm{NaHCO}_{3}(150 \mathrm{ml})$ was added to the reaction mixture under ice-cooling. After the mixture was vigorously stirred for $10 \mathrm{~min}$, it was extracted with AcOEt $(250 \mathrm{ml} \times 2)$, which was washed with water, dried over anhydrous $\mathrm{Na}_{2} \mathrm{SO}_{4}$, and concentrated in vacuo. The residue was subjected to column chromatography to give $8 \mathbf{a}-\mathbf{c}$. Yields of $\mathbf{8 a}-\mathbf{c}$ are summarized in Table 1.

Di-tert-butyl 3-Dimethylphosphono-1,2,3,6-tetrahydropyridazine-1,2dicarboxylate (8a) Colorless prisms (hexane), mp $65-65.5^{\circ} \mathrm{C} .{ }^{1} \mathrm{H}-\mathrm{NMR}$ $\left(\mathrm{CDCl}_{3}\right) \delta: 1.48,1.49$ and $1.50^{13)}\left(18 \mathrm{H}\right.$, each s, $\left.\mathrm{C}_{4} \mathrm{H}_{9} \times 2\right), 3.61-3.93(7 \mathrm{H}$, $\mathrm{m}, \mathrm{OCH}_{3} \times 2$ and $\left.\mathrm{C} 6-\mathrm{Ha}\right), 4.34$ and $4.48^{13)}(1 \mathrm{H}, \mathrm{dd}, J=18.0,7.0 \mathrm{~Hz}$ and dd, $J=16.5,6.6 \mathrm{~Hz}, \mathrm{C} 6-\mathrm{Hb}), 4.82-5.25(1 \mathrm{H}, \mathrm{br}, \mathrm{C} 3-\mathrm{H}), 5.95$ and $5.98^{13)}(2 \mathrm{H}$, each brs, C4-H, C5-H). ${ }^{13} \mathrm{C}-\mathrm{NMR}\left(\mathrm{CDCl}_{3}\right) \delta: 28.14,28.19,28.23$ (q), $41.17,43.18(\mathrm{t}), 50.60\left(\mathrm{dd},{ }^{1} J \mathrm{CP}=160.8 \mathrm{~Hz}, \mathrm{C} 3\right), 52.96\left(\mathrm{qd},{ }^{2} J \mathrm{CP}=6.1 \mathrm{~Hz}\right.$, $\left.\mathrm{OCH}_{3}\right), 53.48\left(\mathrm{qd}^{2} J \mathrm{CP}=6.1 \mathrm{~Hz}, \mathrm{OCH}_{3}\right), 53.84\left(\mathrm{qd},{ }^{2} J \mathrm{CP}=6.1 \mathrm{~Hz}, \mathrm{OCH}_{3}\right)$, $80.75,82.23$ (s), 120.51, 120.78 (d), 126.00 (d), 152.09, 153.73, 154.28 (s). IR $(\mathrm{KBr}) \mathrm{cm}^{-1}$ : 1708. MS m/z: $392\left(\mathrm{M}^{+}\right)$. Anal. Calcd for $\mathrm{C}_{16} \mathrm{H}_{29} \mathrm{~N}_{2} \mathrm{O}_{7} \mathrm{P}: \mathrm{C}$, 48.98; H, 7.45; N, 7.14. Found: C, 48.75; H, 7.26; N, 7.12.

Bis(2,2,2-trichloroethyl) 3-Dimethylphosphono-1,2,3,6-tetrahydropyridazine-1,2-dicarboxylate (8b) Colorless oil. ${ }^{1} \mathrm{H}-\mathrm{NMR}\left(\mathrm{CDCl}_{3}\right) \delta: 3.80$ $\left(3 \mathrm{H}, \mathrm{d}, J \mathrm{HP}=11.0 \mathrm{~Hz}, \mathrm{OCH}_{3}\right), 3.82\left(3 \mathrm{H}, \mathrm{d}, J \mathrm{HP}=11.0 \mathrm{~Hz}, \mathrm{OCH}_{3}\right), 3.87-$ 3.97 and $4.02-4.12^{13)}(1 \mathrm{H}, \mathrm{br}, \mathrm{C} 6-\mathrm{Ha}), 4.45-5.26\left(6 \mathrm{H}, \mathrm{m}, \mathrm{COOCH}_{2} \times 2\right.$, $\mathrm{C} 3-\mathrm{H}, \mathrm{C} 6-\mathrm{Hb}), 5.98-6.11(2 \mathrm{H}, \mathrm{br}, \mathrm{C} 4-\mathrm{H}, \mathrm{C} 5-\mathrm{H}) .{ }^{13} \mathrm{C}-\mathrm{NMR}\left(\mathrm{CDCl}_{3}\right) \delta$ : $42.74,43.75(\mathrm{t}), 51.41,51.62\left(\mathrm{dd},{ }^{1} J \mathrm{CP}=161.8 \mathrm{~Hz}, \mathrm{C} 3\right), 53.23(\mathrm{qd}$, $\left.{ }^{2} J \mathrm{CP}=6.1 \mathrm{~Hz}, \mathrm{OCH}_{3}\right), 54.17\left(\mathrm{qd}^{2} J \mathrm{CP}=6.1 \mathrm{~Hz}, \mathrm{OCH}_{3}\right), 75.50,75.62,75.88$ (t), 94.64, 94.90, 95.07 (s), 119.93, 120.37 (d), 124.97, 125.46 (d), 151.99, 153.04, 153.87 (s). IR (neat) $\mathrm{cm}^{-1}: 1728$. MS $m / z: 540\left(\mathrm{M}^{+}\right)$. HR-MS $m / z$ : $539.8796\left(\mathrm{M}^{+}\right)\left(\right.$Calcd for $\left.\mathrm{C}_{12} \mathrm{H}_{15} \mathrm{Cl}_{6} \mathrm{~N}_{2} \mathrm{O}_{7} \mathrm{P}: 539.8748\right)$.

Dibenzyl 3-Dimethylphosphono-1,2,3,6-tetrahydropyridazine-1,2-dicarboxylate (8c) Colorless oil. ${ }^{1} \mathrm{H}-\mathrm{NMR}\left(\mathrm{CDCl}_{3}\right) \delta: 3.38-3.99(7 \mathrm{H}, \mathrm{m}$, $\mathrm{OCH}_{3} \times 2$ and $\left.\mathrm{C} 6-\mathrm{Ha}\right), 4.41-4.62(1 \mathrm{H}, \mathrm{m}, \mathrm{C} 6-\mathrm{Hb}), 4.93-5.33(5 \mathrm{H}, \mathrm{m}$, $\mathrm{COOCH}_{2} \times 2$ and $\left.\mathrm{C} 3-\mathrm{H}\right), 5.97(2 \mathrm{H}$, br s, C4-H, C5-H), 7.15-7.43 (10H, m, $\left.\mathrm{C}_{6} \mathrm{H}_{5} \times 2\right) .{ }^{13} \mathrm{C}-\mathrm{NMR}\left(\mathrm{CDCl}_{3}\right) \delta: 42.06,43.29,43.38(\mathrm{t}), 51.19(\mathrm{dd}$, $\left.{ }^{1} J \mathrm{CP}=154.1 \mathrm{~Hz}, \mathrm{C} 3\right), 52.93,53.35,53.81,54.20(\mathrm{q}), 67.93,67.99,68.56$, $68.62,68.69$ (t), 120.01, 120.43 (d), 125.23, 125.71 (d), 127.82, 127.88, 127.94, 128.05, 128.20, 128.29, 128.35, 128.49, 128.55 (d), 135.40, 135.93, 136.07 (s), 153.61, 154.84, 155.34 (s). IR (neat) $\mathrm{cm}^{-1}: 1716$. MS $\mathrm{m} / \mathrm{z}: 460$ $\left(\mathrm{M}^{+}\right)$. HR-MS $m / z$ : $460.1399\left(\mathrm{M}^{+}\right)\left(\right.$Calcd for $\left.\mathrm{C}_{22} \mathrm{H}_{25} \mathrm{~N}_{2} \mathrm{O}_{7} \mathrm{P}: 460.1400\right)$.

One-Pot Reaction Including the Hetero-D-A Reaction and Phosphonylation Trimethyl phosphite $(3.10 \mathrm{~g}, 25 \mathrm{mmol})$ was added under ice-cooling to a solution of 1-substituted 1,3-butadiene $5(11 \mathrm{mmol})$, di-tert-butyl azodicarboxylate $6 \mathrm{a}(2.30 \mathrm{~g}, 10 \mathrm{mmol})$ in $\mathrm{CH}_{2} \mathrm{Cl}_{2}(10 \mathrm{ml})$ beforehand stirred for $30 \mathrm{~min}$. A solution of $\mathrm{BF}_{3} \cdot \mathrm{Et}_{2} \mathrm{O}(2.13 \mathrm{~g}, 15 \mathrm{mmol})$ in $\mathrm{CH}_{2} \mathrm{Cl}_{2}(40 \mathrm{ml})$ was 
added slowly to the reaction mixture under ice-cooling. The reaction mixture was allowed to warm to room temperature and stirred for a further $12 \mathrm{~h}$. The work-up was carried out in a manner similar to that described above for the phosphonylation of 7. The crude product 8a was recrystallized from hexane. Yields of 8a from different butadienes $\mathbf{5 a}-\mathbf{c}$ are summarized in Table 1.

Di-tert-butyl 3-Dimethylphosphono-hexahydropyridazine-1,2-dicarboxylate (9a) A solution of $8 \mathbf{a}(4.70 \mathrm{~g}, 12 \mathrm{mmol})$ in methanol $(100 \mathrm{ml})$ was hydrogenated over $10 \% \mathrm{Pd}-\mathrm{C}(0.3 \mathrm{~g})$ at $4 \mathrm{~atm}$ and room temperature for $20 \mathrm{~h}$. The reaction mixture was filtered and concentrated under reduced pressure to leave a crude product, which was subjected to column chromatography $\left(\mathrm{CHCl}_{3}\right.$, and then $\left.3 \% \mathrm{MeOH}-\mathrm{CHCl}_{3}\right)$ to give 9a. Yield $89 \%$. Colorless oil. ${ }^{1} \mathrm{H}-\mathrm{NMR}\left(\mathrm{CDCl}_{3}\right) \delta$ : 1.48 and 1.49 (each $\left.9 \mathrm{H}, \mathrm{s}, \mathrm{C}_{4} \mathrm{H}_{9} \times 2\right), 1.73-2.23$ $\left(4 \mathrm{H}, \mathrm{m}, \mathrm{C} 4-\mathrm{H}_{2}, \mathrm{C} 5-\mathrm{H}_{2}\right), 2.83-3.08(1 \mathrm{H}, \mathrm{m}, \mathrm{C} 6-\mathrm{Ha}), 3.71-3.94(6 \mathrm{H}, \mathrm{m}$, $\left.\mathrm{OCH}_{3} \times 2\right), 3.79-4.22(1 \mathrm{H}, \mathrm{m}, \mathrm{C} 6-\mathrm{Hb}), 4.43-4.84(1 \mathrm{H}, \mathrm{br}, \mathrm{C} 3-\mathrm{H}) .{ }^{13} \mathrm{C}-$ $\operatorname{NMR}\left(\mathrm{CDCl}_{3}\right) \delta: 19.99(\mathrm{t}), 22.59(\mathrm{t}), 28.22(\mathrm{q}), 42.69,45.02(\mathrm{t}), 47.74(\mathrm{dd}$, $\left.{ }^{1} J \mathrm{CP}=159.8 \mathrm{~Hz}, \quad \mathrm{C} 3\right), \quad 52.50 \quad\left(\mathrm{qd},{ }^{2} J \mathrm{CP}=7.4 \mathrm{~Hz}, \mathrm{OCH}_{3}\right), 52.74 \quad(\mathrm{qd}$, $\left.{ }^{2} J \mathrm{CP}=6.1 \mathrm{~Hz}, \mathrm{OCH}_{3}\right), 80.32,81.81(\mathrm{~s}), 152.55,153.92,154.49,154.87(\mathrm{~s})$. IR $(\mathrm{KBr}) \mathrm{cm}^{-1}: 1712$. MS m/z: $394\left(\mathrm{M}^{+}\right)$. HR-MS m/z: $394.1869\left(\mathrm{M}^{+}\right)$ (Calcd for $\mathrm{C}_{16} \mathrm{H}_{31} \mathrm{~N}_{2} \mathrm{O}_{7} \mathrm{P}: 394.1869$ ).

Bis(2,2,2-trichloroethyl) 3-Dimethylphosphono-hexahydropyridazine1,2-dicarboxylate (9b) A solution of $\mathbf{8 b}(6.48 \mathrm{~g}, 12 \mathrm{mmol})$ in methanol $(100 \mathrm{ml})$ was hydrogenated over $10 \% \mathrm{Pd}-\mathrm{C}(0.3 \mathrm{~g})$ at $1 \mathrm{~atm}$ and room temperature for $20 \mathrm{~h}$. The work-up of the mixture was carried out in a manner similar to that described above for 9a. Yield $40 \%$. Colorless prisms (isopropyl ether), mp $90-90.5^{\circ} \mathrm{C} .{ }^{1} \mathrm{H}-\mathrm{NMR}\left(\mathrm{CDCl}_{3}\right) \delta: 1.56-1.70(1 \mathrm{H}, \mathrm{br}$, $\mathrm{C} 4-\mathrm{Ha}), 1.89-2.31\left(3 \mathrm{H}, \mathrm{m}, \mathrm{C} 4-\mathrm{Hb}, \mathrm{C} 5-\mathrm{H}_{2}\right), 3.05-3.18$ and $\left.3.22-3.33^{13}\right)$ $\left(1 \mathrm{H}\right.$, each m, C6-Ha), $3.80\left(3 \mathrm{H}, \mathrm{d}, J \mathrm{HP}=11.0 \mathrm{~Hz}, \mathrm{OCH}_{3}\right), 3.85(3 \mathrm{H}, \mathrm{d}$, $\left.J \mathrm{HP}=11.0 \mathrm{~Hz}, \mathrm{OCH}_{3}\right), 4.22-4.34(1 \mathrm{H}, \mathrm{m}, \mathrm{C} 3-\mathrm{H}), 4.40-4.54(1 \mathrm{H}, \mathrm{br}, \mathrm{C} 6-$ $\mathrm{Hb}), 4.59-5.25\left(4 \mathrm{H}, \mathrm{m}, \mathrm{COOCH}_{2} \times 2\right) .{ }^{13} \mathrm{C}-\mathrm{NMR}\left(\mathrm{CDCl}_{3}\right) \delta: 19.52,19.84$ (t), 22.50, $22.60(\mathrm{t}), 44.55,45.81(\mathrm{t}), 48.98\left(\mathrm{dd},{ }^{1} J \mathrm{CP}=161.8 \mathrm{~Hz}, \mathrm{C} 3\right), 49.22$ $\left(\mathrm{dd},{ }^{1} J \mathrm{CP}=163.3 \mathrm{~Hz}, \mathrm{C} 3\right), 53.00\left(\mathrm{qd},{ }^{2} \mathrm{JCP}=7.6 \mathrm{~Hz}, \mathrm{OCH}_{3}\right), 53.94(\mathrm{qd}$, $\left.{ }^{2} \mathrm{JCP}=7.6 \mathrm{~Hz}, \mathrm{OCH}_{3}\right), 75.36,75.56,75.76(\mathrm{t}), 94.85,95.07,95.23(\mathrm{~s})$, 152.38, 153.14, 154.20 (s). IR (KBr) cm ${ }^{-1}: 1753,1724$. MS m/z: $542\left(\mathrm{M}^{+}\right)$, Anal. Calcd for $\mathrm{C}_{12} \mathrm{H}_{17} \mathrm{Cl}_{6} \mathrm{~N}_{2} \mathrm{O}_{7} \mathrm{P}$ : C, 26.45; H, 3.14; N, 5.14. Found: C, 26.57; H, 3.05; N, 5.17 .

Hexahydropyridazine-3-phosphonic Acid (Piperidazine-3-phosphonic Acid) (3) A solution of 9a $(789 \mathrm{mg}, 2 \mathrm{mmol})$ in $6 \mathrm{~N} \mathrm{HCl}(20 \mathrm{ml})$ was refluxed under an argon atmosphere for $12 \mathrm{~h}$ to give 3 as $\mathrm{HCl}$ salt after evapo- ration to dryness, which was dissolved in $\mathrm{MeOH}(3 \mathrm{ml})$ and treated with propylene oxide $(1 \mathrm{ml}, 14 \mathrm{mmol})$. The precipitate that resulted was collected by filtration and recrystallized from $\mathrm{MeOH}$-propylene oxide $(3: 1)$ to give 3 in $62 \%$ yield. White powder (MeOH-propylene oxide), $\mathrm{mp} 158-160^{\circ} \mathrm{C}$. ${ }^{1} \mathrm{H}-\mathrm{NMR}\left(\mathrm{D}_{2} \mathrm{O}\right) \delta: 1.56-1.85(2 \mathrm{H}, \mathrm{m}, \mathrm{C} 4-\mathrm{Ha}, \mathrm{C} 5-\mathrm{Ha}), 1.90-2.05(2 \mathrm{H}, \mathrm{m}$, $\mathrm{C} 4-\mathrm{Hb}, \mathrm{C} 5-\mathrm{Hb}), 3.03-3.12(1 \mathrm{H}, \mathrm{m}, \mathrm{C} 6-\mathrm{Ha}), 3.16-3.26(1 \mathrm{H}, \mathrm{m}, \mathrm{C} 3-\mathrm{H})$, $3.30-3.40(1 \mathrm{H}, \mathrm{m}, \mathrm{C} 6-\mathrm{Hb}),{ }^{13} \mathrm{C}-\mathrm{NMR}\left(\mathrm{D}_{2} \mathrm{O}\right) \delta: 21.30\left(\mathrm{td},{ }^{3} J \mathrm{CP}=12.2 \mathrm{~Hz}\right.$, C5), 23.72 (t, C4), $46.04(\mathrm{t}, \mathrm{C} 6), 54.88\left(\mathrm{dd},{ }^{1} \mathrm{JCP}=146.5 \mathrm{~Hz}, \mathrm{C} 3\right)$. IR (KBr) $\mathrm{cm}^{-1}$ : 3400, $3255(\mathrm{OH}, \mathrm{NH}), 1155(\mathrm{P}=\mathrm{O}), 1061(\mathrm{P}-\mathrm{O}) . \mathrm{MS} m / z: 167$ $\left(\mathrm{M}^{+}+1\right)$. HR-MS $m / z$ : $167.0586\left(\mathrm{M}^{+}+1\right)$ (Calcd for $\mathrm{C}_{4} \mathrm{H}_{12} \mathrm{~N}_{2} \mathrm{O}_{3} \mathrm{P}$ : 167.0586).

Acknowledgment This work was supported in part by the Special Research Fund of Hokuriku University.

\section{References and Notes}

1) Diel P. J., Maier L., Eur. Pat. Appl. EP 143078 (1985) [Chem. Abstr., 103, $15544 m$ (1985)].

2) Rachon J., Wasielewski C., Rocz. Chem., 50, 477-487 (1976).

3) Diel P. J., Maier L., Phosphorus and Sulfur, 36, 85-98 (1988).

4) Heydari A., Javidan A., Schaffie M., Tetrahedron Lett., 42, 80718073 (2001).

5) Yuan C., Chen S., Xie R., Feng H., Maier L., Phosphorus Sulfur Silicon, 106, 115-123 (1995).

6) Yuan C., Li C., Synthesis, 1996, 507-510 (1996).

7) Kaname M., Yoshinaga K., Arakawa Y., Yoshifuji S., Tetrahedron Lett., 40, 7993-7994 (1999).

8) Bevan K., Davies J. S., Hassall C. H., Morton R. B., Phillips D. A. S., J. Chem. Soc. C, 1971, 514-521 (1971).

9) Smitka T. A., Deeter J. B., Hunt A. H., Mertz F. P., Ellis R. M., Boeck L. D., Yao R. C., J. Antibiot., 41, 726-733 (1988).

10) Alder K., Niklas H., Liebig's Ann. Chem., 585, 81-96 (1954).

11) Snyder, H. R., Jr., Michels J. G., J. Org. Chem., 28, $1144-1146$ (1963).

12) Langlois N., Rojas-Rousseau A., Decavallas O., Tetrahedron Asymmetry, 7, 1095-1100 (1996).

13) These signals were split due to the rotamers and/or the conformers. 